\section{MATERNAL AND PERINATAL OUTCOMES OF CORONAVIRUS DISEASE COVID 19 INFECTION IN PREGNANCY - A RETROSPECTIVE STUDY.}

KEY WORDS: COVID 19 maternal outcomes, perinatal outcomes, APGAR score, vertical transmission

\section{Dr Richa}

Udhwani

Dr Shiny

Varghese*
DNB Resident.

Senior Consultant. *Corresponding Author

Dr Maria Cecilia Senior Consultant.

Purpose: To study maternal and perinatal outcomes of COVID-positive pregnant women in a tertiary healthcare centre.

Materials And Methods: This was a retrospective statistical analysis of records of COVID positive women who delivered at our institution between May 2020 to December 2020.

Results: There were 44 COVID positive pregnant women during the study period of which $40(90.9 \%)$ were term pregnancies and $4(9.09 \%)$ preterm pregnancies. There were $17(38.63 \%)$ women who had spontaneous onset of labour, $14(31.8 \%)$ were induced and 13(29.5\%) had elective LSCS. 14(31.8\%) women had normal vaginal delivery,26(59.09\%) had LSCS and 4(9.09\%) had instrumental delivery. Most women 39(88.6\%) were asymptomatic and $5(11.3 \%)$ presented with mild symptoms. 30 patients $(68.18 \%)$ had underlying medical disorders. There were 5 $(11.3 \%)$ babies with low birth weight $(<2.5 \mathrm{~kg})$, APGAR scores at 5 min was $>7$ for all babies. Six $(15.7 \%)$ babies required NICU admission and $2(4.5 \%)$ were COVID positive.

Conclusion: Majority of the COVID positive women were asymptomatic. Majority of the patients had an underlying comorbidity.None underwent ICU admission. Risk of preterm delivery and NICU admission was similar to COVID negative patients. Risk of vertical transmission exists. Fetal outcomes in terms of APGAR scores at 5 min, birth weight was found to be good.

\section{INTRODUCTION:}

The severe acute respiratory syndrome coronavirus 2 (SARSCoV-2) occurring due to exposure of the susceptible population to COVID virus has led to a global crisis. Pregnant women are comparatively more susceptible to severe infections, due to the physiological changes that occur in pregnancy (1).Pregnancy is associated with alterations in cell mediated immunity. Therefore pregnant women have increased susceptibility to infection by intracellular organisms such as viruses (2). However studies have concluded that COVID infection may not present as a severe disease in pregnant women. Hence it is essential to determine the effect of COVID on pregnant women to ensure better control of the disease and to allow caregivers and policy makers to suggest recommendations. (3). It is important that caregivers are aware of the spectrum of presentations and outcomes of COVID-19 infection during pregnancy and childbirth as there may be deleterious effects of COVID on brain development and function due to dysregulation of factors such as cytokines and the complement cascade that may occur in COVID 19 (4). Another important concern is the vertical transmission (5). Hence it is essential that pregnant women and their new-borns, being potential risk groups in the current global pandemic, are evaluated. After review of literature it was found that data on the maternal and perinatal outcomes of pregnant women who were infected with the SARS-CoV-2 are limited as there are very few case reports and series having small sample sizes with diverse findings. Therefore this study is being conducted in a tertiary health care center to determine the effect of the global pandemic on pregnant women and their new-borns.

\section{Methods:}

This was a retrospective analysis of data collected from records of covid positive pregnant women who delivered in Bangalore Baptist hospital,Bangalore from May 2020December 2020. The demographics of the women were noted along with covid symptoms if any.Severity of the disease, any associated risk factors, mode of delivery and post natal |www.worldwidejournals.com complications were noted. Neonatal outcomes such as birth weight, apgar scores at 5 minutes, covid status of the baby and need for NICU care was noted down. Statistical analysis was done.

\section{RESULTS:}

There were a total 44 women included in the study. Mean age of presentation was 29 years (21 -39 years ). Out of which $11(25 \%)$ were primigravida and $33(75 \%)$ were multigravida. Out of the primigravida $2(18.1 \%)$ patients had instrumental delivery, $1(9 \%)$ had normal vaginal delivery 8 (72.7\%)underwent LSCS. Out of the multigravida ,13(39.3\%) had normal vaginal delivery, $2(6 \%)$ had instrumental delivery, 18(54.5\%) had LSCS.

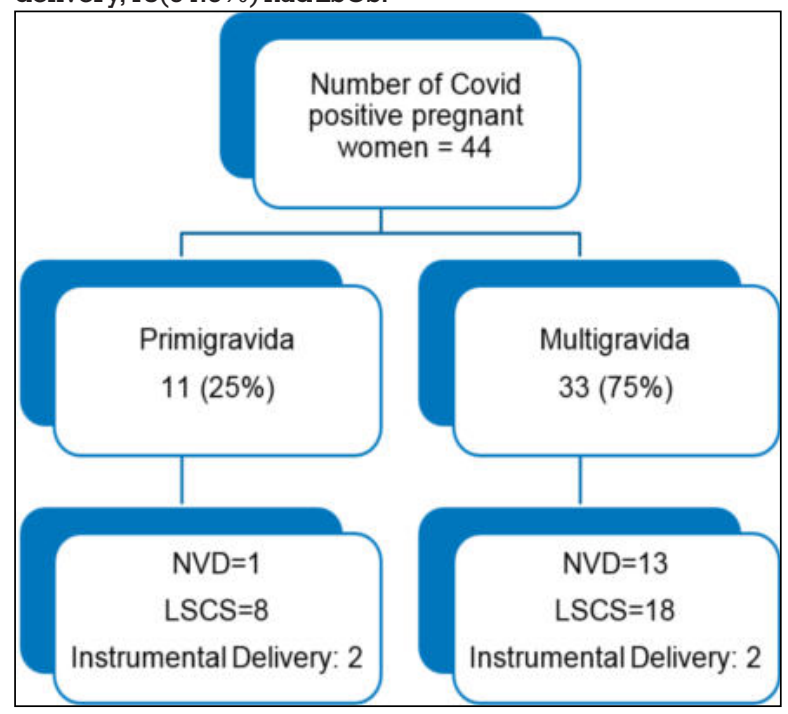

Figure 1: Mode of delivery.

Out of the total pregnancies only $4(9 \%)$ were preterm deliveries ,rest of the $40(90 \%)$ patients had term deliveries. There were 17 (38.63\%) women who had spontaneous onset 
of labour, 14 (31.8\%) women who were induced and $13(29.5 \%)$ women had elective LSCS. Among the women who had spontaneous onset of labour $7(41.1 \%)$ women had vaginal delivery (3(42\%) normal, $2(28 \%)$ forceps and $1(14 \%)$ vacuum delivery) and 10(58.8\%) had emergency LSCS. Among those who were induced, $11(78.5 \%)$ a vaginal delivery (10 (90\%) normal and $1(9 \%)$ (vacuum delivery) and 3 $(21.4 \%)$ had emergency LSCS.

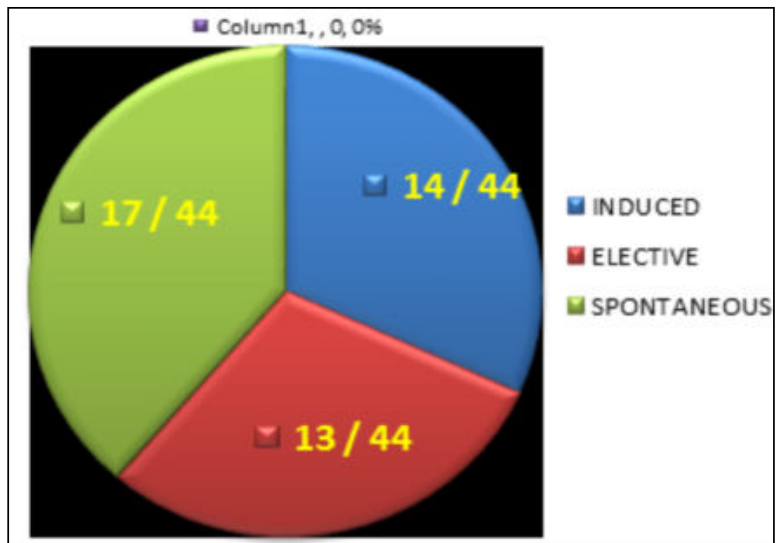

Figure 2 : Onset Of Labour.

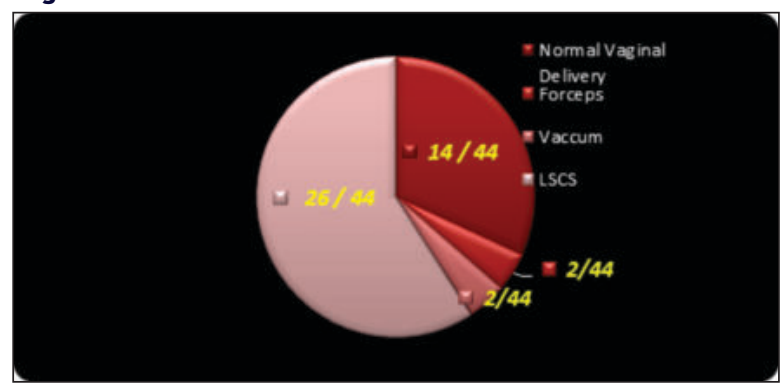

Figure 3 : Maternal Outcomes In Terms OfType Of Deliveries

Most women (35/38)(92\%) were asymptomatic for the covid disease and the other 5/44(11.3\%) presented with mild symptoms. Among these 44 women, 14(31.8\%) had no underlying medical disorders, $18(40.9 \%)$ had gestational diabetes mellitus, $7(15.9 \%)$ had subclinical hypothyroidism, $2(4.5 \%)$ had anemia, $2(4.5 \%)$ with bronchial asthma , $2(4.5 \%)$ with gestational hypertension, 1 (2.2\%) with HIV.

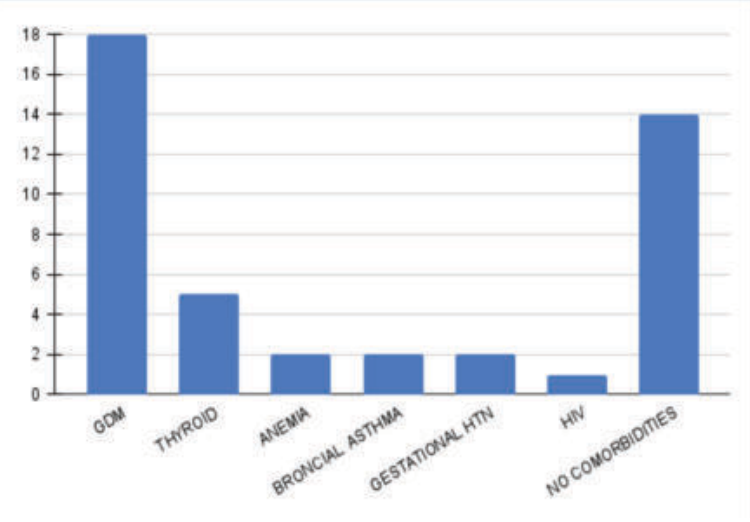

Figure 4 : Underlying Co Morbidity In COVID Positive Women.

There were 26 LSCS , 12(46.1\%) emergency LSCS, 14(53.8\%) elective LSCS. Indication was fetal distress for $4(15.3 \%)$ patient , failure of induction for $2(7.6 \%)$ patients, maternal request for 6 (23.07\%)patients, previous LSCS for $7(26.9 \%)$ patients and others $(26.9 \%)$ for rest of the patients. COVID disease per se is not an indication for LSCS, Indication are similar to COVID negative women.

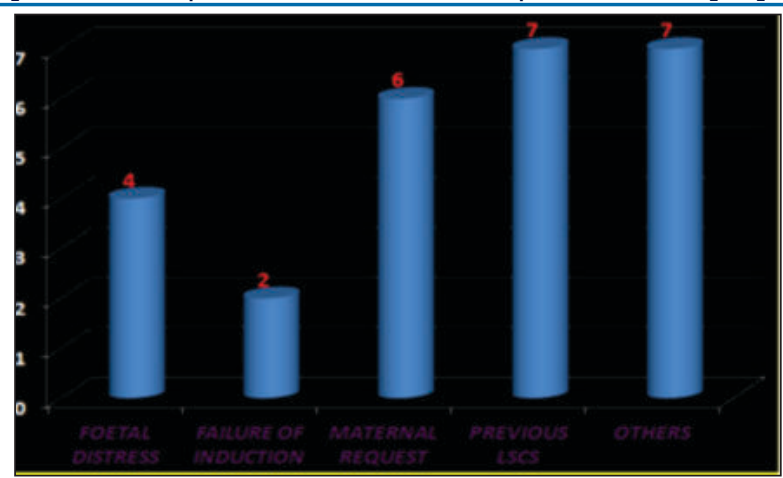

Figure 5 : Indications For LSCS.

There were 5 (11.3\%) babies with low birth weight $(<2.5 \mathrm{~kg})$ but APGAR scores at 5 min was $>7$ for all babies . 6 (15.7\%) babies required NICU admission but none had any prolonged NICU stay or complications. Out of 44 babies, 2 (4.5\%) were COVID positive, but both remained asymptomatic without any sequelae.

\section{DISCUSSION :}

In our study we found that most patient were asymptomatic and had an underlying co morbidity, but none of the women had severe morbidity or needed ICU admission and most patients had term delivery. Risk of vertical transmission was present, but fetal outcomes were good in terms of baby weight and APGAR scores. In a systematic review and metaanalysis published in British Medical Journal(6) concluded that : Pregnant women may less likely manifest covid-19 related symptoms like fever or myalgia than non-pregnant women of reproductive age and may need intensive care treatment for covid-19. Pregnant women in hospital with covid-19 are less likely to show symptoms than non-pregnant women of similar age but may have an increased risk of admission to intensive care.Pre-existing co morbidities like advanced maternal age , along with high body mass index seem to be risk factors for severe covid-19. Preterm birth rates are high in pregnant women with covid-19 as compared to pregnant women without the disease.

In another systemic review and meta-analysis published in American journal of obstetrics and gynaecology(7): concluded that preterm delivery is the most common adverse pregnancy outcome. COVID-19 infection was associated with increased rate of preterm birth, preeclampsia, cesarean, and perinatal death.There have been no published cases indicating risk of vertical transmission, although the findings should be interpreted with caution in view of the very small number of included cases.

In the Royal College of Obstetricians and Gynaecologist (RCOG) guidelines for COVID in pregnancy it was concluded that pregnant women are at not at a greater risk of becoming seriously unwell. Majority of the pregnant women will experience only mild or moderate symptoms. There is no evidence to suggest there is an increased risk of miscarriage.Vertical transmission does occur, but it is uncommon. Federation of Obstetric and Gynaecological Societies of India (FOGSI) in its guidelines also concluded that : Pregnant women are not likely to be severely unwell. They may experience only mild or moderate cold flu like symptoms. If the pregnant woman has co morbid conditions they are more likely to have a severe form of respiratory disease. The infection is not associated with risk of vertical transmission and there is no risk of abortion, although risk of preterm exists. Results of our study are consistent with most of available studies, systematic reviews, meta-analysis and published literature.

\section{CONCLUSION :}

Majority of the COVID positive patients were asymptomatic 
or presented with mild symptoms. Induction of labour can be considered as an option in COVID positive patients. Majority of the patients had an underlying comorbidity.None of the patients underwent ICU admission or had a severe morbidity. Risk of preterm delivery was not greater as compared to COVID negative patients in our study. Risk of vertical transmission cannot be ruled out, e. Fetal outcomes in terms of APGAR scores at $5 \mathrm{~min}$, birth weight were good.

\section{Conflict Of Interest Statement :}

The author declares that there is no conflict of interest.

\section{Funding :}

Authors received no finiancial support for the study.

\section{Disclaimer :}

The views expressed in the submitted article are the own words of the authors and not an official position of the institution.

\section{REFERENCES :}

1. Goodnight WH, Soper DE. Pneumonia in pregnancy. Crit Care Med. 2005;33:S390-S397

2. Nelson-Piercy C. Respiratory disease. In: Handbook of Obstetric Medicine. Boca Raton:CRC Press; 2015:37 l p

3. Mor G, Cardenas I. The immune system in pregnancy: $A$ unique com-plexity. Am J Reprod Immunol. 2010;63:425-433

4. Tsafaras GP, Ntontsi P, Xanthou G. Advantages and limitations of the neonatal immune system. Front Pediatr. 2020;8:5

5. Chan GJ, Lee AC, Baqui AH, Tan J, Black RE. Risk of early-onset neonatal infection with maternal infection or colonization: a global systematic review and meta-analysis. PLoS Med.2013;10:e100150

6. Mahase E. Covid-19: Pregnant women with virus are more likely to need intensive care, study finds.

7. Breslin N, Baptiste C, Miller R, Fuchs K, Goffman D, Gyamfi-Bannerman C, D'Alton M. Coronavirus disease 2019 in pregnancy: early lessons. American journal of obstetrics \& gynecology MFM.2020 May 1;2(2):10011 l. 